\title{
JORDAN DERIVATIONS ON SEMIPRIME RINGS
}

\author{
M. BRESAR
}

(Communicated by John B. Conway)

\begin{abstract}
I. N. Herstein has proved that any Jordan derivation on a 2torsion free prime ring is a derivation. In this paper we prove that Herstein's result is true in 2-torsion free semiprime rings. This result makes it possible for us to prove that any linear Jordan derivation on a semisimple Banach algebra is continuous, which gives an affirmative answer to the question posed by $\mathbf{A}$. M. Sinclair in [5].
\end{abstract}

Preliminaries. Throughout this paper all rings will be associative. Let $R$ be a ring. The center of $R$ will be denoted by $Z(R)$. We shall write $[a, b]$ for $a b-b a$. A ring $R$ is said to be 2-torsion free, if whenever $2 a=0$, with $a \in R$, then $a=0$. A ring $R$ is called a prime ring if $a R b=(0)$ implies $a=0$ or $b=0$. A ring $R$ is called a semiprime ring if $a R a=(0)$ implies $a=0$. Let $R$ be any ring. An additive mapping ' $: R \rightarrow R$ is called a derivation if $(a b)^{\prime}=a^{\prime} b+a b^{\prime}$ holds for all pairs $a, b \in R$. An additive mapping ' $: R \rightarrow R$ is called a Jordan derivation if $\left(a^{2}\right)^{\prime}=a^{\prime} a+a a^{\prime}$ holds for all $a \in R$. Obviously, every derivation is a Jordan derivation. The converse is, in general, not true. A well-known result of I. N. Herstein [2] states that every Jordan derivation on a 2-torsion free prime ring is a derivation. A brief proof of this result can be found in [1]. The main purpose of this paper is to present a generalization of Herstein's result. More precisely, we shall prove that every Jordan derivation on a 2-torsion free semiprime ring is a derivation. In particular, every Jordan derivation on a 2-torsion free semisimple ring is a derivation, which generalizes a result of $\mathrm{A}$. M. Sinclair (see [5]). From the fact that every linear derivation on a semisimple Banach algebra is continuous, and from our generalization of Herstein's result, it follows immediately that every Jordan derivation on a semisimple Banach algebra is continuous, which gives an affirmative answer to the question posed by A. M. Sinclair in [5]. In the last part of the paper two characterizations of 2-torsion free prime rings are obtained.

The results. As we have mentioned above, the main purpose of this paper is to prove the following result.

THEOREM 1. Let $R$ be a 2-torsion free semiprime ring and let ': $R \rightarrow R$ be a Jordan derivation. In this case, ${ }^{\prime}$ is a derivation.

For the proof of Theorem 1 we need several steps. We begin with the proposition below.

Received by the editors December 7, 1987.

1980 Mathematics Subject Classification (1985 Revision). Primary 16A12, 16A68, 16A72, 46H99.

Key words and phrases. Prime ring, semiprime ring, semisimple Banach algebra, derivation, Jordan derivation.

This work was supported by the Research Council of Slovenia. 
PROPOSITION 2. Let $R$ be a 2-torsion free ring and let ': $R \rightarrow R$ be a Jordan derivation. Then for all $a, b, c \in R$ the following statements hold:

$$
(a b+b a)^{\prime}=a^{\prime} b+a b^{\prime}+b^{\prime} a+b a^{\prime}
$$

$$
(a b a)^{\prime}=a^{\prime} b a+a b^{\prime} a+a b a^{\prime}
$$

$$
(a b c+c b a)^{\prime}=a^{\prime} b c+a b^{\prime} c+a b c^{\prime}+c^{\prime} b a+c b^{\prime} a+c b a^{\prime} .
$$

The proof of Proposition 2 is entirely elementary and can be found in [2] and [3]. Let ' be any Jordan derivation. We follow Herstein [2] and write $a^{b}$ for $(a b)^{\prime}-a^{\prime} b-a b^{\prime}$. It is easy to see that for all $a, b, c \in R$

$$
a^{b+c}=a^{b}+a^{c}, \quad(a+b)^{c}=a^{c}+b^{c}
$$

hold. Let us point out that the statement (i) in Proposition 2 can be written in the form

$$
a^{b}=-b^{a} .
$$

The theorem below can be found in [1] but we shall present the proof for the sake of completeness.

THEOREM 3. Let $R$ be a 2-torsion free ring and let ': $R \rightarrow R$ be a Jordan derivation. In this case for all elements $a, b, x \in R$

$$
a^{b} x[a, b]+[a, b] x a^{b}=0
$$

holds.

ProOF. Consider $W=(a b x b a+b a x a b)^{\prime}$. Using (i) in Proposition 2 we obtain

$$
\begin{aligned}
W= & (a(b x b) a)^{\prime}+(b(a x a) b)^{\prime} \\
= & a^{\prime} b x b a+a(b x b)^{\prime} a+a b x b a^{\prime}+b^{\prime} a x a b+b(a x a)^{\prime} b+b a x a b^{\prime} \\
= & a^{\prime} b x b a+a b^{\prime} x b a+a b x^{\prime} b a+a b x b^{\prime} a+a b x b a^{\prime}+b^{\prime} a x a b+b a^{\prime} x a b \\
& +b a x^{\prime} a b+b a x a^{\prime} b+b a x a b^{\prime} .
\end{aligned}
$$

On the other hand, according to (iii) in Proposition 2 we see that

$$
\begin{aligned}
W & =((a b) x(b a)+(b a) x(a b))^{\prime} \\
& =(a b)^{\prime} x b a+a b x^{\prime} b a+a b x(b a)^{\prime}+(b a)^{\prime} x a b+b a x^{\prime} a b+b a x(a b)^{\prime} .
\end{aligned}
$$

By comparing and using (i) in Proposition 2 we obtain (3).

We continue with the lemma which might be of some interest beyond its application to the study of Jordan derivations on semiprime rings.

LEMMA 4. Let $R$ be a 2-torsion free semiprime ring and let $a, b \in R$. If for all $x \in R$ the relation

$$
a x b+b x a=0
$$

holds, then $a x b=b x a=0$ is fulfilled for all $x \in R$.

PROOF. Let $x$ and $y$ be arbitrary elements from $R$. Using (4) three times we obtain $[a(x) b] y a x b=-[b(x a y) a] x b=a x[a(y) b] x b=-a x b y a x b$. Thus $2(a x b) y(a x b)=$ 0 for all $x, y \in R$. Since $R$ is a 2-torsion free semiprime ring, it follows that $a x b=0$ for all $x \in R$. 
Proof OF THEOREM 1. Our aim is to show that $a^{b}=0$ for all $a, b \in R$. From Theorem 3 and Lemma 4 it follows immediately that

$$
a^{b} x[a, b]=0
$$

for all $a, b, x \in R$. According to (1) a linearization of (5) with regard to $b$ gives $a^{b} x[a, c]+a^{c} x[a, b]=0$. Using this relation and (5) we obtain $\left(a^{b} x[a, c]\right) y\left(a^{b} x[a, c]\right)$ $=-a^{b} x[a, c] y a^{c} x[a, b]=0$ for all $a, b, c, x, y \in R$. Since $R$ is semiprime we have

$$
a^{b} x[a, c]=0
$$

for all $a, b, c, x \in R$. A linearization of (6) gives $a^{b} x[d, c]+d^{b} x[a, c]=0$. Hence $\left(a^{b} x[d, c]\right) y\left(a^{b} x[d, c]\right)=-a^{b} x[d, c] y d^{b} x[a, c]=0$ by (6). Thus

$$
a^{b} x[d, c]=0
$$

for all $a, b, c, d, x \in R$. In particular, $\left[a^{b}, c\right] x\left[a^{b}, c\right]=\left(a^{b} c-c a^{b}\right) x\left[a^{b}, c\right]=a^{b}(c x)\left[a^{b}, c\right]$ $-c a^{b}(x)\left[a^{b}, c\right]=0$ for all $a, b, c, x \in R$. Using again the fact that $R$ is semiprime we may conclude that $\left[a^{b}, c\right]=0$ for all $a, b, c \in R$. In other words, we have proved that $a^{b} \in Z(R)$ for all pairs $a, b \in R$. Using (7) we obtain $\left(a^{b}[d, c]\right) x\left(a^{b}[d, c]\right)=0$, hence

$$
a^{b}[d, c]=0
$$

for all $a, b, c, d \in R$. According to (2) we have $2\left(a^{b}\right)^{2}=a^{b}\left(a^{b}-b^{a}\right)=a^{b}\left((a b)^{\prime}-\right.$ $\left.(b a)^{\prime}+\left[b^{\prime}, a\right]+\left[b, a^{\prime}\right]\right)$. By $(8), a^{b}\left[b^{\prime}, a\right]=0$ and $a^{b}\left[b, a^{\prime}\right]=0$, and so the relation above reduces to

$$
2\left(a^{b}\right)^{2}=a^{b}[a, b]^{\prime} .
$$

By (8), $a^{b}[a, b]=0$. Since $a^{b} \in Z(R)$, we have $a^{b}[a, b]+[a, b] a^{b}=0$. Hence, according to (i) in Proposition 2, one obtains that $\left(a^{b}\right)^{\prime}[a, b]+a^{b}[a, b]^{\prime}+[a, b]^{\prime} a^{b}+$ $[a, b]\left(a^{b}\right)^{\prime}=0$. By comparing this relation with (9) and using the fact that $a^{b} \in$ $Z(R)$ we arrive at

$$
4\left(a^{b}\right)^{2}+\left(a^{b}\right)^{\prime}[a, b]+[a, b]\left(a^{b}\right)^{\prime}=0
$$

where $a$ and $b$ are arbitrary elements from $R$. If we multiply (10) by $a^{b}$ we obtain $4\left(a^{b}\right)^{3}=0$ for all $a, b \in R$, since (8) holds. By the assumption $R$ is 2-torsion free and so we have $\left(a^{b}\right)^{3}=0$. It is easy to see that the center of a semiprime ring does not contain any nonzero nilpotents. Therefore we can conclude that $a^{b}=0$ for all $a, b \in R$. The proof of the theorem is complete.

COROLLARY 5. Let $R$ be a 2-torsion free semisimple ring and let ': $R \rightarrow R$ be a Jordan derivation. In this case' is a derivation.

PROOF. A consequence of Theorem 1 and of the fact that every semisimple ring is semiprime.

In [5] A. M. Sinclair has proved that every continuous linear Jordan derivation on a semisimple Banach algebra is a derivation. The corollary above is a generalization of Sinclair's result. We conclude our discussion on Jordan derivations with the following result. 
THEOREM 6. Let $A$ be a Banach algebra and let ': $A \rightarrow A$ be a linear Jordan derivation. In this case' is continous.

Proof. B. E. Johnson and A. M. Sinclair [4] have proved that every linear derivation on a semisimple Banach algebra is continuous. Therefore, using their result and Corollary 5 we are forced to conclude that Theorem 6 holds.

As we have mentioned above Theorem 6 gives an affirmative answer to Sinclair's question which can be found in [5]. The following result characterizes 2 -torsion free prime rings among all 2-torsion free rings.

THEOREM 7. Let $R$ be a 2-torsion free ring. Then the following conditions are equivalent:

(i) $R$ is a prime ring.

(ii) Let $a, b \in R$ and let $a x b+b x a=0$ for all $x \in R$. Then $a=0$ or $b=0$.

(iii) Let $a, b \in R$ and let $a x a=b x b$ for all $x \in R$. Then $a=b$ or $a=-b$.

PROOF. (i) $\Leftrightarrow($ ii). The implication (i) $\Rightarrow$ (ii) is the assertion of Lemma 3.10 in [3]. On the other hand, it is an immediate consequence of Lemma 4. Let condition (ii) hold. Suppose that $a x b=0$ for all $x \in R$. Then $(b x a) y(b x a)+(b x a) y(b x a)=$ $2 b x(a y b) x a=0$ for all $x, y \in R$ which yields $b x a=0$ for all $x \in R$. Hence $a x b+b x a=0$ for all $x \in R$ and therefore $a=0$ or $b=0$. Thus $R$ is prime.

(ii) $\Leftrightarrow$ (iii). Let condition (ii) hold and let $a x a=b x b$ for all $x \in R$. Then $(a-b) x(a+b)+(a+b) x(a-b)=0$ for all $x \in R$ which yields $a-b=0$ or $a+b=0$. The implication (ii) $\Rightarrow$ (iii) is therefore proved. Let condition (iii) hold and suppose that $a x b+b x a=0$ for all $x \in R$. Consequently, $(a-b) x(a-b)=(a+b) x(a+b)$ for all $x \in R$. Thus $a-b=a+b$ or $a-b=-(a+b)$. Since $R$ is 2 -torsion free we have $a=0$ or $b=0$ which completes the proof.

It should be mentioned that for the proofs of implications (ii) $\Rightarrow(\mathrm{i})$ and (ii) $\Rightarrow$ (iii) we did not use the assumption that $R$ is 2-torsion free.

ACKNOWLEDGEMENT. I wish to express my thanks to Professor J. Vukman for helpful suggestions and for drawing my attention to [4 and 5].

\section{REFERENCES}

1. M. Bresar and J. Vukman, Jordan derivations on prime rings, Bull. Austral. Math. Soc. 37 (1988), 321-322.

2. I. N. Herstein, Jordan derivations of prime rings, Proc. Amer. Math. Soc. 8 (1957), 1104-1110.

3. __ Topics in ring theory, Univ. of Chicago Press, Chicago, London, 1969.

4. B. E. Johnson and A. M. Sinclair, Continuity of derivations and a problem of Kaplansky, Amer. J. Math. 90 (1968), 1067-1073.

5. A. M. Sinclair, Jordan homomorphisms and derivations on semisimple Banach algebras, Proc. Amer. Math. Soc. 24 (1970), 209-214.

Institute of Mathematics, Physics And Mechanics, P. O. Box 543, 61001 LJublJANA, YUGOSLAVIA 